\title{
A Phenomenological Model for Linear Viscoelasticity of Monodisperse Linear Polymers
}

\author{
Kwang Soo Cho*, Woo Sik Kim, Dong-ho Lee, Lee Soon Park, Kyung Eun Min, \\ Kwan Ho Seo, Inn-kyu Kang, and Soo-Young Park \\ Department of Polymer Science, Kyungpook National University, Daegu 701-702, Korea \\ Youngdon Kwon \\ Department of Textile Engineering, Syungkyunkwan University, Suwon 440-746, Korea
}

Received June 25, 2002; Revised Sept. 27, 2002

\begin{abstract}
Although the reptational model of Doi and Edwards gives a successful description of viscoelasticity of flexible linear polymers, the success is restricted to the terminal region. ${ }^{1}$ There have been several attempts to modify the Doi-Edwards model to describe wider range of time or frequency. ${ }^{2-6}$ This paper suggests a simple phenomenological model which can describe wider range of molecular weight than such molecular models can. Although our model is a phenomenological one, it is practical and convenient to predict the effect of molecular weight distribution on linear viscoelastic data because of its simple mathematical form.
\end{abstract}

Keywords : monodisperse polymers, linear viscoelasticity, molecular weight.

\section{Introduction}

Even in linear region of deformation, most polymers show very complex viscoelastic behavior that prohibits simple modeling with a few parameters. Such complexity may result from molecular weight distribution (MWD) of polymer. Thus, the first step of understanding linear viscoelasticity (LVE) of polymers must be to understand LVE of monodisperse polymers. The second step may be to know the LVE of their mixtures. The last step may be to determine the MWD from LVE of polydisperse polymers.

Before the Doi- Edward (DE) model $^{7}$ appeared, the generalized Maxwell (GM) model seems to have been one of the most efficient way of modeling LVE of polymers. In case of the GM approach, the most interesting theme is to determine or model the relaxation time distribution (RTD). Determination of the RTD from the LVE implies to solve an integral equation, which is known as an ill-posed problem. ${ }^{8}$ Although the GM model gives very accurate curve fitting, it is not a convenient way when one wants to connect LVE with MWD.

The DE theory achieved outstanding success in LVE of polymers. It explains molecular weight dependency of viscoelastic functions. However, the theory is restricted to the

*e-mail : polphy@knu.ac.kr

1598-5032/10/266-07 (C)2002 Polymer Society of Korea terminal region of entangled polymer melts. In the DE theory, the plateau modulus $G_{N}^{(0)}$ is independent of molecular weight and is the common scale factor of viscoelastic functions such as the zero shear viscosity $\eta_{0}$, the steady-state compliance $J_{e}^{(0)}$ and so on.

There have been several attempts to modify the DE model to fit entire range of LVE. Since all these theories are based on the DE model, the scale factor of the moduli is the plateau modulus that is difficult to be determined when molecular weight is not larger than about ten times of the entanglement molecular weight $M_{e}$. This implies that any model scaled by the plateau modulus suffers from difficulty in extension to low molecular weights where the plateau is not clear.

To the knowledge of authors, Lins model $^{2}$ was the first successful modification of the DE model. Although the model was for the relaxation modulus of polymers with very narrow MWD, in curve fitting, it used discrete MWD with 3 peaks, which must be different from a real MWD.

Benallal et $a l .^{3}$ developed a molecular model as a simple sum of the reptation and the Rouse modes, contour length fluctuation, and the glassy mode. Although the model describes well the LVE of monodisperse polymers with various molecular weights for wide range of frequency, there is a considerable discrepancy in the loss modulus after the terminal region. Since the model is also based on the DE model, the dynamic and the relaxation moduli are scaled by the plateau modulus. 
Milner and McLeish ${ }^{4}$ did another remarkable molecular modeling, which is also a modification of the DE model. In this model, there is no discrepancy in the loss modulus, different from the model of Benallal et al. Furthermore, their approach is applicable to star polymers as well as linear polymers. However, since their model is also a modification of the DE model, the authors did not compare their model with the LVE of the molecular weight range $10 M_{e}>M>M_{e}$.

Chung and coworkers ${ }^{5}$ also contributed in solving the discrepancy of the original model of Doi and Edwards at high frequency based on slip-link model. Their work is to improve the process $\mathrm{X}$ introduced by Lin with more theoretical basis.

Larson and coworkers ${ }^{6}$ did more precise quantitative prediction of LVE for not only monodisperse polymers but also their mixtures. Their approach is a numerical integration of the modified diffusion equation of the reptation. The relaxation modulus is the sum of the Rouse mode from analytical calculation and the reptation mode from the numerical integration. Although their prediction was very precise, all moduli are scaled by the plateau modulus.

In this paper, we aim to develop a simple phenomenological model for monodisperse polymer, which can cover the molecular weight range of $M>M_{e}$. Its simple mathematical form is efficient in application to the prediction of MWD from LVE of polydisperse polymers.

\section{Theory}

The Limit of Molecular Models. The most important success of the DE model is to predict the molecular weight dependence of the zero shear viscosity $\eta_{0}$ for entangled polymer melts. The theory reads ${ }^{7}$

$$
\eta_{0}=\frac{\pi^{2}}{12} G_{N}^{(0)} \tau_{d}
$$

and

$$
\tau_{d} \propto M^{3.4}
$$

where $G_{N}^{(0)}$ is the plateau modulus, $\tau_{d}$ is the disentanglement time and $M$ is molecular weight. Eq. (1) agrees well with experimental data for $M \gg M_{e}$. The DE theory ${ }^{7}$ explains the LVE of monodisperse polymer by the two characteristic times $\tau_{d}$ and $\tau_{e}$. The second characteristic time $\tau_{e}$ indicates the start of the plateau of the relaxation or the storage modului. Since the model assumes the existence of the plateau modulus and uses it as the common scale factor of the zero shear viscosity, dynamic moduli, and the steady-state compliance $J_{e}^{(0)}$, the model is not valid when the plateau modulus cannot be determined. In other words, this theory cannot be applied to the monodisperse polymers without fully developed entanglement. From the help of the experimental data of Schausbergers et al., ${ }^{1}$ one can recognize that the plateau is not clear when $M<10 M_{e}$. In order to use LVE of monodisperse polymer for the prediction of those of polydisperse polymers, a candidate model must describe LVE of the monodisperse polymers of $M<10 M_{e}$.

The Boltzmann superposition principle reads an exact relation between the zero shear viscosity and the relaxation modulus $G(t)$ :

$$
\eta_{0}=\int_{0}^{\infty} G(t) d t
$$

Figure 1 shows schematic behavior of the relaxation moduli of monodisperse polymers. The area [a] in Figure 1 can be approximated by Eq. (1). When $M>10 M_{e}$, the plateau can be defined even though the plateau is not perfectly flat. However, when $M<10 M_{e}$, the two characteristic times $\tau_{d}$ and $\tau_{e}$ are so close that the plateau may not be defined clearly. Thus, when $M<10 M_{e}$, the integration Eq. (3) cannot be approximated by Eq. (1) because the area [b] in Figure 1 is comparable with the area [a] even though the plateau modulus determined from the LVE of polymers with $M>10 M_{e}$, is used. Thus, it is necessary to replace the plateau modulus by more generalized scale factor.

The More Generalized Scale Factors of Modulus. To develop a phenomenological model for monodisperse polymers, the experimental data of Schausbergers et al. ${ }^{1}$ are chosen since they provide tabulated data as well as graphs. They measured LVE of nearly monodisperse polystyrenes with wide range of molecular weights. The reference temperature is $180^{\circ} \mathrm{C}$. Their data are redrawn in Figure 2. The molecular weights of the samples in Figure 2 are shown in Table I.

From the Boltzmann superposition principle, the zero shear viscosity and the steady-state compliance can be obtained from the dynamic moduli ${ }^{7}$ :

$$
\eta_{0}=\lim _{\omega \rightarrow 0} \frac{G^{\prime \prime}(\omega)}{\omega}
$$

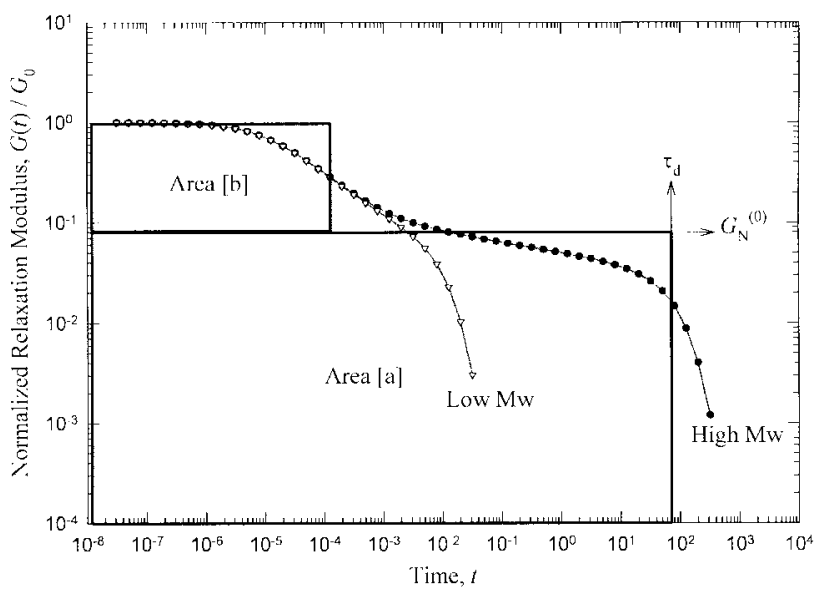

Figure 1. Schematic representation of the relaxation modulus for various molecular weights. 
$\eta_{0}^{2} J_{e}^{(0)}=\lim _{\omega \rightarrow 0} \frac{G^{\prime}(\omega)}{\omega^{2}}$

Since in the terminal region

$$
\begin{aligned}
& G^{\prime}(\omega) \propto \omega^{2} \\
& G^{\prime \prime}(\omega) \propto \omega
\end{aligned}
$$

it is convenient to define new viscoelastic functions such as

$$
\begin{aligned}
& g^{\prime}(\omega) \equiv \frac{G^{\prime}(\omega)}{\omega^{2}} \\
& g^{\prime \prime}(\omega) \equiv \frac{G^{\prime \prime}(\omega)}{\omega}=\eta^{\prime}(\omega)
\end{aligned}
$$

From the Eqs. (4), (5) and (6), it follows that

$$
\begin{aligned}
& \lim _{\omega \rightarrow 0} g^{\prime}(\omega) \equiv A_{G}=J_{e}^{(0)} \eta_{0}^{2} \\
& \lim _{\omega \rightarrow 0} g^{\prime \prime}(\omega)=\eta_{0}
\end{aligned}
$$

As shown in Figure 2, the storage and the loss moduli do not have simple forms. However, they have locally power-law type behavior. In Figure 2, one can see that at high frequency, the storage moduli of various molecular weights

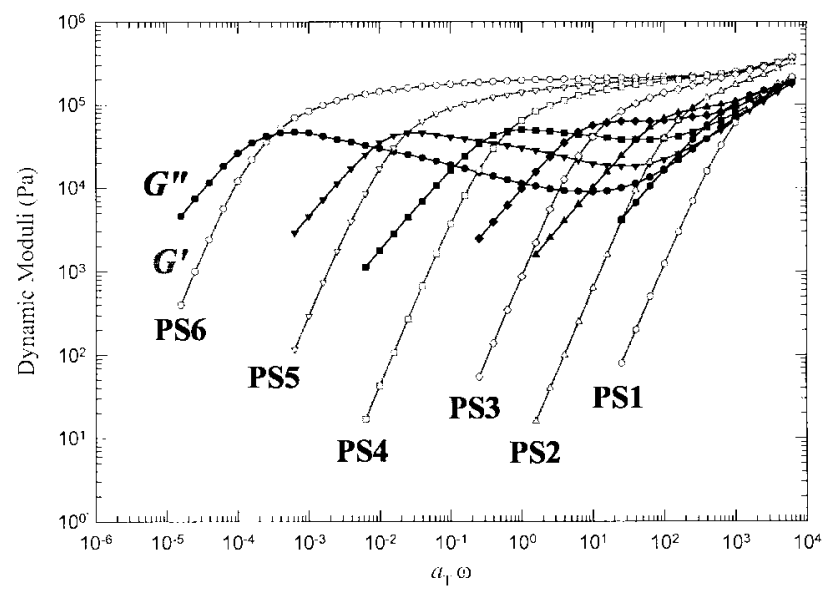

Figure 2. Data of Schausbergers et al. ${ }^{1}$ The storage (open symbols) and the loss (solid symbols) moduli of polystyrene. The reference temperature is $180^{\circ} \mathrm{C}$. Samples are specified in Table I.

Table I. Data of Molecular Weights (Schausberger et al. ${ }^{1}$ )

\begin{tabular}{crc}
\hline Sample & \multicolumn{1}{c}{$M_{w}$} & $M_{w} / M_{n}$ \\
\hline PS1 & 34,000 & 1.05 \\
PS2 & 65,000 & 1.02 \\
PS3 & 125,000 & 1.05 \\
PS4 & 292,000 & 1.09 \\
PS5 & 757,000 & 1.09 \\
PS6 & $2,540,000$ & 1.13 \\
\hline
\end{tabular}

look like a single function of frequency. In the terminal region, the dynamic moduli of different molecular weights look like having similar frequency dependency if they are horizontally shifted according to molecular weight. This becomes clearer in the loss modulus. This implies that LVE of monodisperse polymers seems to be, at least, the sum of two functions of frequency. The one represents the reptational mode which can be superposed by a horizontal shifting factor dependent upon molecular weight and the other represents the glassy mode.

If the data of Schausbergers et al. are redrawn as the plots of $g^{\prime}(\omega) / A_{G}$ and $g^{\prime \prime}(\omega) / \eta_{0}$ against $\omega$, then the plots look like

$$
\begin{aligned}
& \frac{g^{\prime}(\omega)}{A_{G}} \approx \frac{1}{\left(1+t_{d} \omega\right)^{p}} \\
& \frac{g^{\prime \prime}(\omega)}{\eta_{0}} \approx \frac{1}{\left(1+t_{d} \omega\right)^{q}}
\end{aligned}
$$

in the terminal region (Figure 3). Since the exponents $p$ and $q$ can be easily obtained as the slopes of the log-log plots, it is not difficult to determine the characteristic time $t_{d}$ by regression analysis. By the dimensional analysis of $\eta_{0}$,
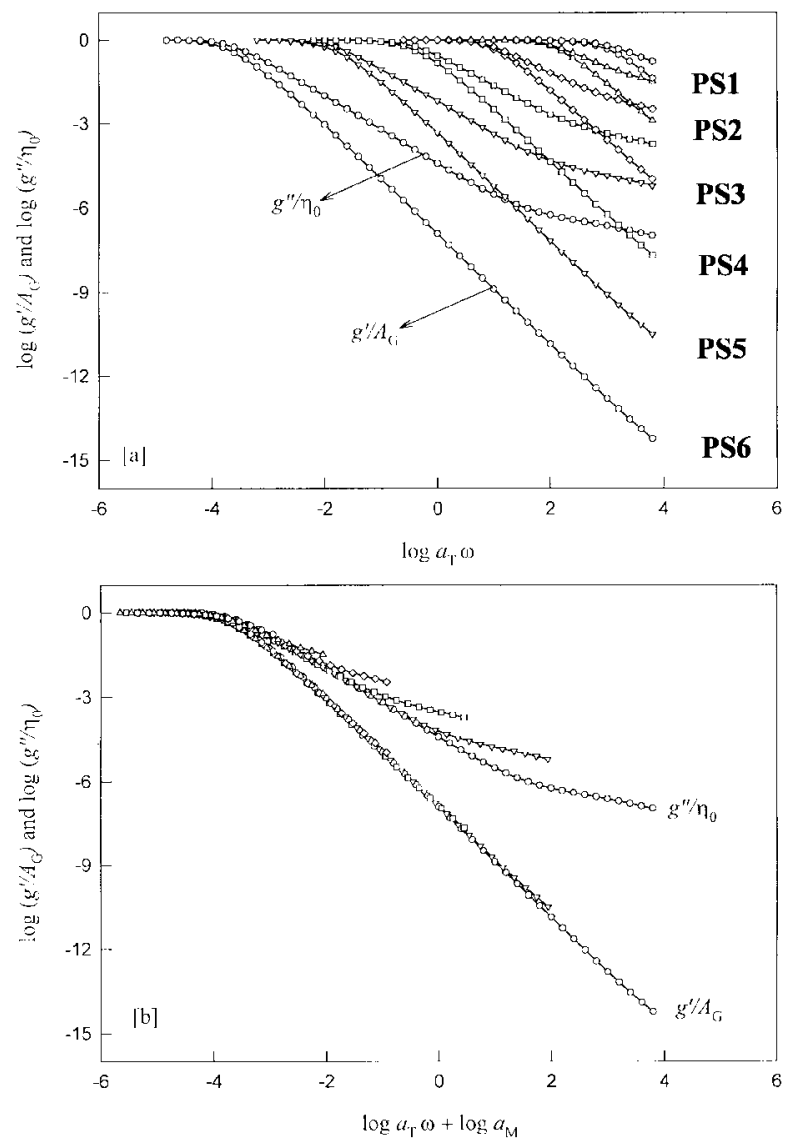

Figure 3. Normalized $g^{\prime}(\omega)$ and $g^{\prime \prime}(\omega)$ (a) and their superposition by molecular weight shifting factor $a_{M}(\mathrm{~b})$. 
$A_{G}$, and $t_{d}$, one can define two moduli which can be used as the scale factors for the dynamic moduli instead of the plateau modulus:

$$
\begin{aligned}
G_{0}{ }^{\prime} & \equiv \frac{A_{G}}{t_{d}^{2}} \\
G_{0}{ }^{\prime \prime} & =\frac{\eta_{0}}{t_{d}}
\end{aligned}
$$

From the help of Eqs. (4) and (5), we have

$$
G_{0}^{\prime}=J_{e}^{(0)} G_{0}^{\prime \prime 2}
$$

Since the terminal behavior Eq. (6) is clear for any molecular weight, the scale factors for modulus, $G_{0}^{\prime}$ and $G_{0}{ }^{\prime \prime}$ can be determined from any experimental result irrespective of molecular weight. Thus, the new scale factors $G_{0}{ }^{\prime}$ and $G_{0}{ }^{\prime \prime}$ are more generalized than the plateau modulus $G_{N}^{(0)}$ in this respect.

The shapes of $g^{\prime}(\omega) / A_{G}$ and $g^{\prime \prime}(\omega) / \eta_{0}$ in Figure 3(a) are almost independent of molecular weight and they can be superposed by a horizontal shift factor. Figure 3(b) is a superposed version of Figure 3(a) where data are horizontally shifted until data of the terminal region are coincided. The superposition of Figure 3(a) makes it clearer that the dynamic moduli are the sum of the two functions, called the reptational and the glassy modes. The branching of $g^{\prime \prime}(\omega) / \eta_{0}$ at higher frequency corresponds to the glassy mode.

The glassy modes of different molecular weights in Figure 3 look like an identical form if they are shifted by an appropriate vertical shifting factor, which is dependent upon molecular weight. This implies that there is another scale factor of modulus for the glassy mode, say $G_{g}$. Thus, we suggest mathematical forms of the dynamic modulus as follows:

$$
\begin{aligned}
& G^{\prime}(\omega)=G_{0}^{\prime} \frac{t_{d}^{2} \omega^{2}}{\left(1+t_{d} \omega\right)^{p}}+G_{g} f^{\prime}(\omega) \\
& G^{\prime \prime}(\omega)=G_{0}^{\prime \prime} \frac{t_{d} \omega}{\left(1+t_{d} \omega\right)^{q}}+G_{g} f^{\prime \prime}(\omega)
\end{aligned}
$$

where $G_{0}{ }^{\prime}, G_{0}{ }^{\prime \prime}$, and $G_{g}$ are the new generalized scale factors which are dependent on molecular weight, and $f^{\prime}(\omega)$ and $f^{\prime \prime}(\omega)$ are functions of frequency to be determined by experimental data. Of course, $f^{\prime}(\omega)$ and $f^{\prime \prime}(\omega)$ are independent of molecular weight, since they represent the glassy mode and all molecular weight dependency are concentrated on $G_{g}$.

The Characteristic Times. At long time scale or in molten state, the main stress contribution originates from the entropy change of chain conformation. However, at short time scale or in glassy state, the main contribution of stress results from interaction energy of the segments in polymer chains. Thus, it is a reasonable viewpoint that there are two relaxation modes according to the origin of stress. One is the relaxation of the entropic stress that may be expressed by the gradient of the conformational entropy with respect to an appropriate strain measure. The other is the relaxation of the energetic stress, called the glassy mode. The former represents the long spatial-range interaction by topological constraints and the latter the short spatial-range interaction between the segments, which includes the intramolecular and the intermolecular interactions.

Thus, from above reasoning, one may think that there are two characteristic time scales for each relaxation mode. The first characteristic time can be chosen as $t_{d}$ which is a function of molecular weight and temperature. It must depend on the reptational diffusion length $L(M, T)$ and the reptational diffusion coefficient $D(M, T)$ :

$$
t_{d}=\frac{L^{2}}{D}
$$

The second characteristic time $t_{0}$ must be independent of molecular weight since it represents the short-range interaction. It must be a function of parameters of the segments such as monomeric friction coefficient and the size of the segments.

Assuming the time temperature superposition (TTS), the two characteristic time scales must have the same temperature dependence, called the temperature horizontal shift factor $a_{T}$. Since the experimental data in Figures 2 and 3 show that both the reptational and the glassy modes of different molecular weights have very similar shape except that the reptational mode is horizontally shifted by molecular weight, it is reasonable assumption that the reptational characteristic time $t_{d}$ consists of two horizontal shift factors $a_{T}$ and $a_{M}$ :

$$
\begin{aligned}
& t_{d}=t_{d}^{r e f} a_{M} a_{T} \\
& t_{0}=t_{0}^{r e f} a_{T}
\end{aligned}
$$

The molecular weight dependency of $a_{M}$ can be determined from experimental data which are shown in Figure 4. In order to guarantee TTS, Eq. (12) must be

$$
\begin{aligned}
& G^{\prime}(\omega)=G_{0}{ }^{\prime} \frac{t_{d}^{2} \omega^{2}}{\left(1+t_{d} \omega\right)^{p}}+G_{g} f^{\prime}\left(t_{0} \omega\right) \\
& G^{\prime \prime}(\omega)=G_{0}^{\prime \prime} \frac{t_{d} \omega}{\left(1+t_{d} \omega\right)^{q}}+G_{g} f^{\prime \prime}\left(t_{0} \omega\right)
\end{aligned}
$$

The characteristic time $t_{0}$ determines the location of the functions $f^{\prime}$ and $f^{\prime \prime}$ in the plots of the dynamic moduli versus frequency.

The Model. Finally, we suggest following model for the dynamic moduli of monodisperse polymers: 


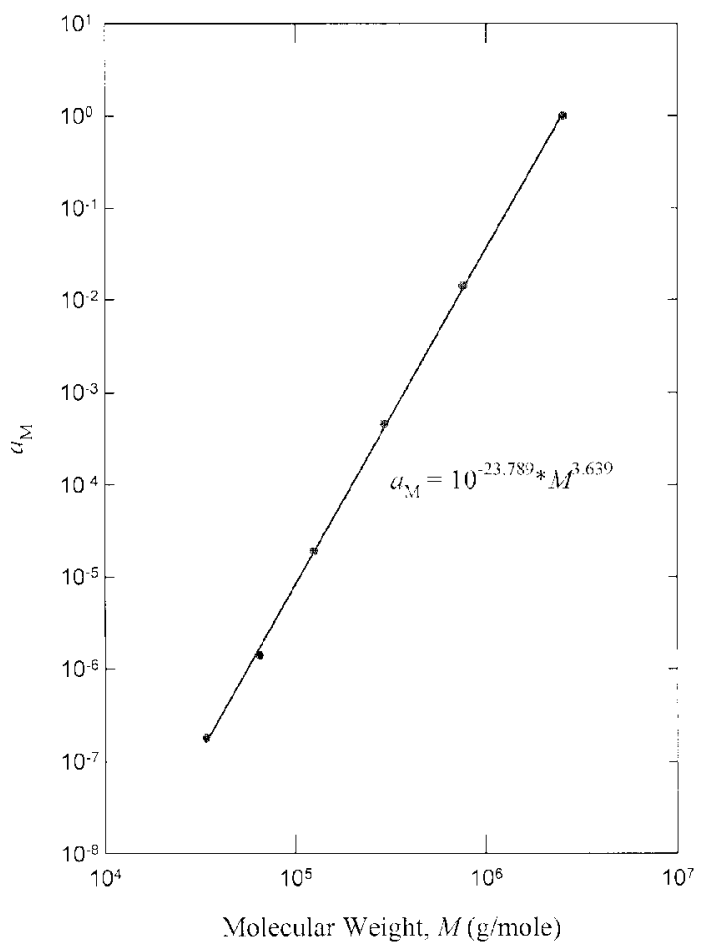

Figure 4. The molecular weight shifting factor $a_{M}$ as a function of molecular weight.

$$
\begin{aligned}
& G^{\prime}(\omega, T)=G_{0} \frac{\left(t_{d} \omega\right)^{2}}{\left(1+t_{d} \omega\right)^{p}}+G_{g} \frac{\left(t_{0} \omega\right)^{m}}{1+\left(t_{0} \omega\right)^{m}} \\
& G^{\prime \prime}(\omega, T)=G_{0}{ }^{\prime \prime} \frac{t_{d} \omega}{\left(1+t_{d} \omega\right)^{q}}+G_{g} \frac{\left(t_{0} \omega\right)^{n}}{1+\left(t_{0} \omega\right)^{m}}
\end{aligned}
$$

The molecular weight dependency of $G_{0}{ }^{\prime}$ and $G_{0}{ }^{\prime \prime}$ can be determined by the slopes of the dynamic moduli in the terminal region, $A_{G}$ and $\eta_{0}$ respectively. Figure 5 shows molecular weight dependencies of $A_{G}$ and $\eta_{0}$. Figure 6 shows the molecular weight dependency of $G_{0}{ }^{\prime}$ and $G_{0}{ }^{\prime \prime}$ when the reference time $t_{d}^{r e f}$ was chosen as the reptational characteristic time of PS6 $\left(t_{d}^{r e f}=3965 \mathrm{sec}\right)$. Since the nature of $G_{0}{ }^{\prime}$ and $G_{0}{ }^{\prime \prime}$ is very similar to that of the plateau modulus, $G_{0}^{\prime}$ and $G_{0}^{\prime \prime}$ become constant as molecular weight increases. On the other hand, both of them increase as molecular weight decreases. Such molecular weight dependency is the same as that of the inverse of the steadystate compliance $J_{e}^{(0)}{ }^{7,9}$ Experimental data of Figure 6 are fitted well by following equations:

$$
\begin{aligned}
G_{0}{ }^{\prime} & =C_{0}{ }^{\prime}+\frac{C_{1}{ }^{\prime}}{1+M / M_{C}} \\
G_{0}{ }^{\prime \prime} & =C_{0}{ }^{\prime \prime}+\frac{C_{1}{ }^{\prime \prime}}{1+M / M_{C}}
\end{aligned}
$$

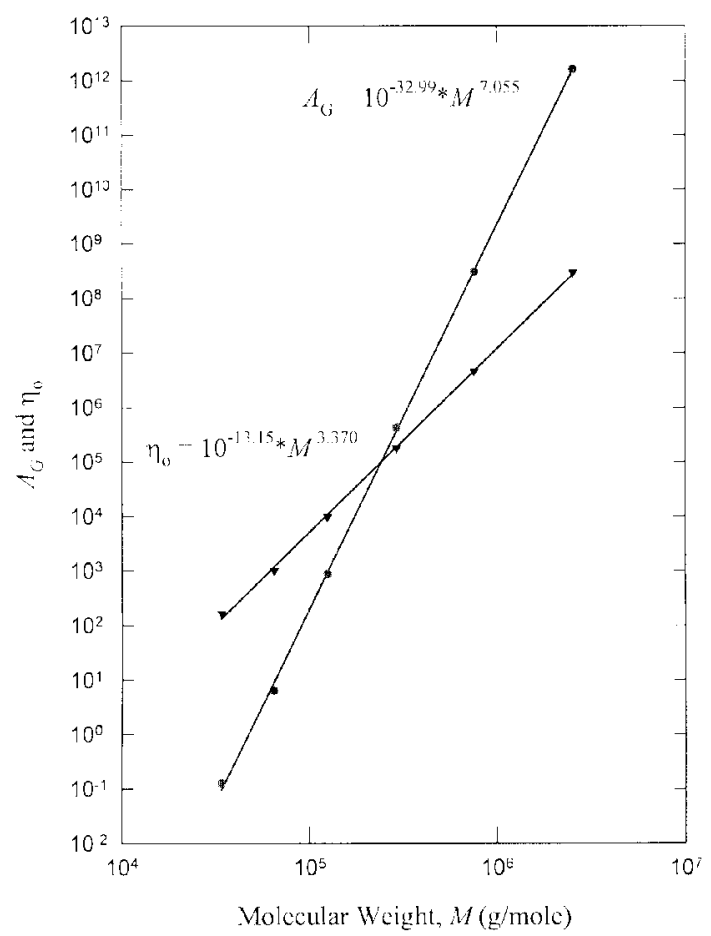

Figure 5. Molecular weight dependency of the zero shear viscosity and $A_{G}$.

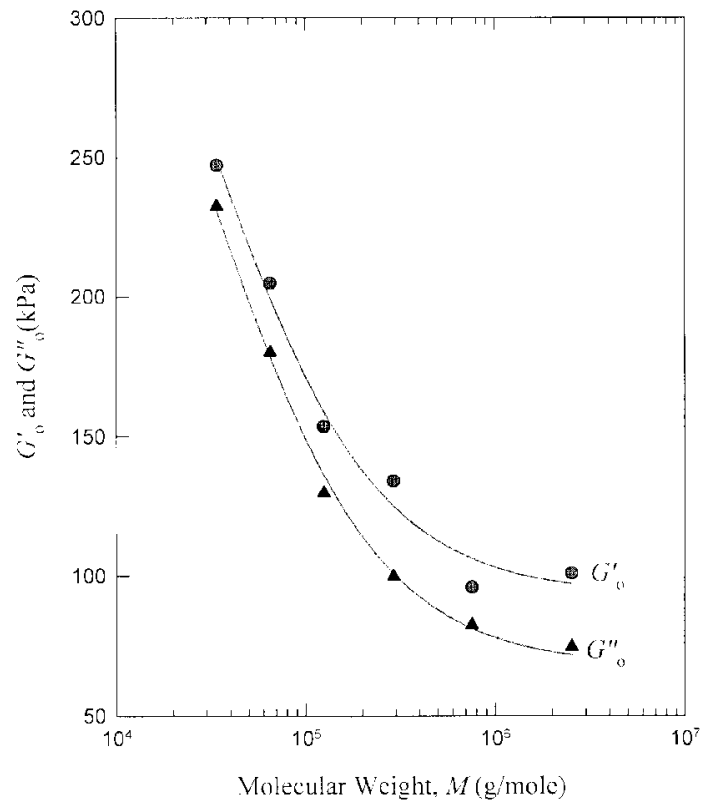

Figure 6. The modulus scale factors for the reptational mode, $G_{0}^{\prime}$ and $G_{0}^{\prime \prime}$ as functions of molecular weight.

where $C_{0}{ }^{\prime}, C_{1}{ }^{\prime}, C_{0}{ }^{\prime \prime}$, and $C_{1}{ }^{\prime \prime}$ are material constant with dimension of modulus and $M_{c}$ is a critical molecular weight.

Differently from $G_{0}{ }^{\prime}$ and $G_{0}{ }^{\prime \prime}$, experimental data are not sufficient to describe molecular weight dependency of $G_{g}$. 
However, most physical properties of polymers increase as molecular weight until certain critical molecular weight and after the critical molecular weight, the physical properties are nearly constant. ${ }^{10}$ Such molecular weight dependency may be approximated by

$$
G_{g}=\frac{G_{\infty}}{1+M_{C} / M}
$$

where $G_{\infty}$ is the glassy modulus at infinite molecular weight, $M_{C}$ is chosen as the same molecular weight in Eq. (17).

\section{Results and Discussion}

To obtain the data of Figure $6, t_{d}$ was determined by nonlinear regression and superposition of Figure 3. The shift factor $a_{M}$ as a function of molecular weight is shown in Figure 4 . The regression of $a_{M}$ with respect to $M$ results in

$$
a_{M}=10^{-23.276} M^{3.639}
$$

Although this result shows that $t_{d}$ is not proportional to $M^{3.4 \pm 0.1}$, the zero shear viscosity determined from $G^{\prime \prime}$ shows (Figure 5)

$$
\eta_{0}=10^{-13.75} M^{3.370}
$$

The regression results Eqs. (19) and (20) are not surprising, because for wide range of molecular weight, the zero shear viscosity is no longer product of the plateau modulus and the maximum relaxation time $\tau_{d}$. In addition, it is difficult to determine the plateau modulus. As shown in Lins experimental data, ${ }^{2}$ the log-log plot of the relaxation modulus versus time, there is a significant slope in the plateau region. Thus the plateau modulus is not well-defined quantity from experimental data compared with $G_{0}{ }^{\prime}$ and $G_{0}{ }^{\prime \prime}$.

Determination of the parameters of the glassy mode, $t_{0}, m$, $n$, and $G_{\infty}$ requires data of higher frequency which are not easily found in literatures. Thus, we consider the parameters of the glassy mode as adjustable parameters. To reduce the number of parameters, a critical molecular weight $M_{C}$ is chosen as about two times of $M_{e}, M_{C}=32,000 \mathrm{~g} / \mathrm{mole}$.

The parameters of curve fitting for the data of Schaus-

Table II. Parameters for Curve Fitting

\begin{tabular}{cccc}
\hline$p$ & 1.93 & $G_{\infty}$ & $7 \times 10^{7} \mathrm{~Pa}$ \\
\hline$q$ & 1.25 & $G_{0}{ }^{\prime}$ & $9.3 \times 10^{4} \mathrm{~Pa}$ \\
\hline$m$ & 1.62 & $G_{1}{ }^{\prime}$ & $3.2 \times 10^{5} \mathrm{~Pa}$ \\
\hline$n$ & 0.62 & $G_{0}{ }^{\prime \prime}$ & $6.7 \times 10^{4} \mathrm{~Pa}$ \\
\hline$t_{d}^{r e f}$ & $3965 \mathrm{sec}$ & $G_{1}{ }^{\prime \prime}$ & $3.4 \times 10^{5} \mathrm{~Pa}$ \\
\hline$t_{0}$ & $10^{-8} \mathrm{sec}$ & $M_{c}$ & $32,000 \mathrm{~g} / \mathrm{mole}$ \\
\hline
\end{tabular}
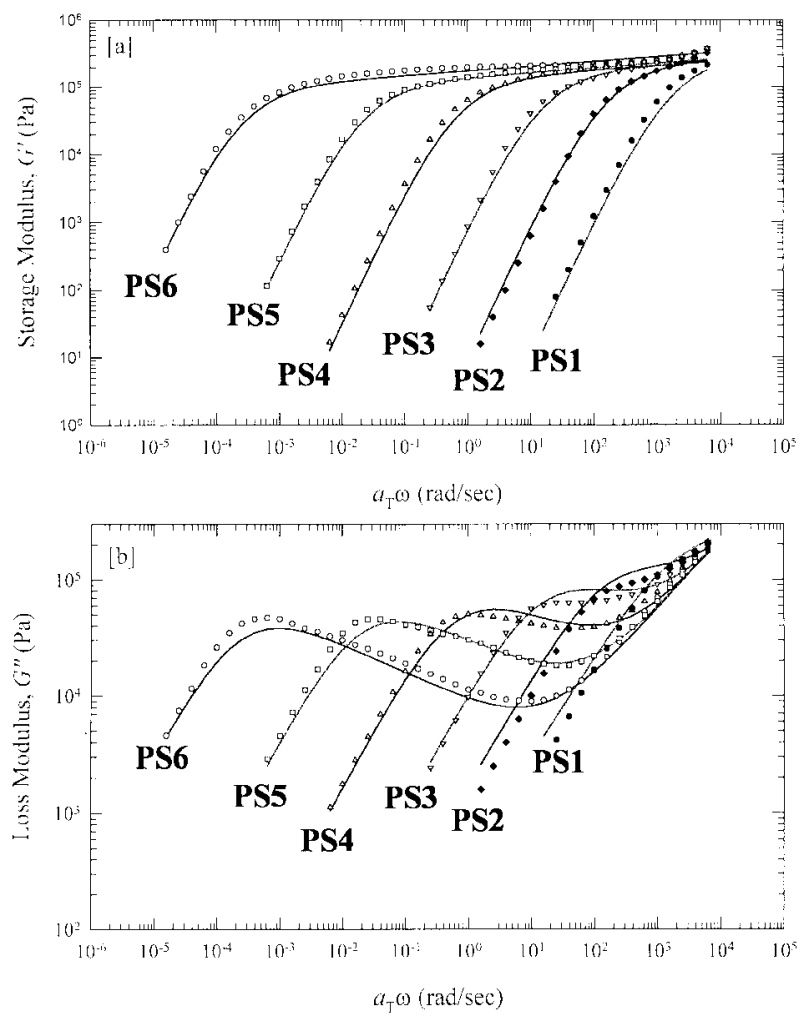

Figure. 7. Comparison of the model with the experimental data of Schausbergers et al. ${ }^{1}$ (a) The storage modulus and (b) the loss modulus.

bergers et al. also are shown in Table II. Usually, all molecular models mentioned above fitted only 3 polystyrene of Schausbergers et al., PS4, PS5, and PS6, whose molecular weights are larger than $10 M_{e}$. It is because the plateau region is well defined for such polymer samples.

Figure 7 shows the curve fitting of the model for the data of Schausbergers et al. The model agrees well with all samples of Schausbergers et al. Thus, the model is expected to be efficient in application to the prediction of MWD from LVE since it gives good curve fitting for wider range of molecular weight with simple mathematical form.

\section{Conclusions}

We develop a phenomenological model for LVE of linear monodisperse polymers, which gives good curve fitting for wider range of molecular weight with simpler mathematical form than the previous molecular models. Instead of the plateau modulus, the model is based on three scale factors of modulus, which are well-defined from experimental data.

The LVE of monodisperse polymer can be described by the sum of two contribution, the reptational and the glassy modes, whose characteristic time scales are $t_{d}$ and $t_{0}$. It is expected that the simple phenomenological model is more efficient in the prediction of MWD from LVE than the 
molecular models.

Acknowledgement. The authors deeply acknowledge for the support of Brain Korea 21.

\section{References}

(1) A. Schausberger, G. Schindlauer, and H. Janeschitz-Kriegl, Rheol. Acta, 24, 220 (1985).

(2) Y.-H. Lin, Macromolecules, 17, 2846 (1984).

(3) A. Benallal, G. Marin, J. P. Montfort, and C. Derail, Macromolecules, 26, 7229 (1993).

(4) S. T. Milner and T. C. B. McLeish, Phys. Rev. Lett., 81, 725
(1998).

(5) K. M. Kim, B. R. Ryu, and I. J. Chung, Nihon Reoroji Gakkaishi, 21, 110 (1993).

(6) C. Pattamprom, R. G. Larson, and T. J. Van Dyke, Rheol. Acta, 39, 517 (2000).

(7) M. Doi and S. F. Edwards, The Theory of Polymer Dynamics, Clarendon Press, Oxford, 1986.

(8) J. Honerkamp and J. Weese, Rheol. Acta, 32, 65 (1993).

(9) J. D. Ferry, Viscoelastic Properties of Polymers $3^{\text {rd }}$ Ed., John Wiley \& Sons, Inc., New York, 1980.

(10) D. W. van Krevelen, Properties of Polymers $2^{\text {nd }}$ Ed., Elsevier, Amsterdam, 1976. 